\title{
La práctica educativa. Análisis desde los mecanismos de influencia educativa y la interacción del profesorado y el estudiantado*
}

\section{Educational practice. Analysis from the mechanisms of educational influence and interaction of teachers and students}

\section{A prática educativa. Análises a partir dos mecanismos de influência educativa $\epsilon$ a interação entre o professorado $\in$ a estudantada}

\author{
Ana Lucía Rosero Prado** \\ CINDE, Universidad de Manizales, Colombia \\ Marco Fidel Chica Lasso*** \\ Universidad de San Buenaventura, Bogotá, Colombia
}

* Artículo de Investigación. Este artículo se deriva de la investigación Mecanismos de influencia educativa en la enseñanza y el aprendizaje de la argumentación en niños y niñas de 5 a 6 años del nivel de transición de la Educación Preescolar. Tesis doctoral. Doctorado en Ciencias Sociales, Niñez y Juventud. Alianza CINDE-Universidad de Manizales, Colombia, 2012.

** Magíster en Educación Especial. Énfasis en Dificultades en el Aprendizaje, Especialista en Docencia Universitaria, Licenciada en Filosofía y Letras, Licenciada en Educación Preescolar. Estudiante del Doctorado en Ciencias Sociales, Niñez y Juventud - de la alianza CINDE-Universidad de Manizales. Miembro del Grupo de Investigación «Educación y pedagogía: saberes, imaginarios e intersubjetividades» - Línea de Investigación «Educación y pedagogía: saberes, imaginarios e intersubjetividades». Maestra Normal Superior Farallones de Cali, Universidad Santiago de Cali y Universidad del Valle, Colombia. E-mail: lucharop83@yahoo.com

*** Doctor en Educación. Magister en Educación y Desarrollo Social, Especialista en Educación Personalizada, Especialista en Gestión Pública, Licenciando en Filosofía y Letras. Docente Investigador del Doctorado en Ciencias Sociales, Niñez y Juventud - de la alianza CINDE-Universidad de Manizales. Miembro del Grupo de Investigación «Educación y pedagogía: saberes, imaginarios e intersubjetividades» - Línea de investigación «Educación y pedagogía: saberes, imaginarios e intersubjetividades». Miembro del Grupo de Investigación «Tendencias Actuales en Educación y Pedagogía» - Línea de Investigación "Antropología Pedagógica y Desarrollo Humano». Decano de la Facultad de Educación de la Universidad de San Buenaventura, Bogotá, Colombia.E-mail: mfchica@usbbog.edu.co 
Resumen. Este artículo hace explícito el sentido y significado de la investigación cualitativa para la vida del ser humano en tanto hace posible que desde los contextos sociales, los actores y sus intersubjetividades interpreten, comprendan y expliquen fenómenos sociales. Considerando la educación como un hecho social, en su interior se abordan los aportes metodológicos y epistemológicos realizados por César Coll con el método denominado «mecanismos de influencia educativa», que robustecen los enfoques socioculturales y contribuyen al análisis de las prácticas educativas, los contextos escolares y dan respuesta específica a cuales son algunos de los mecanismos de influencia educativa que operan en la interactividad entre el profesorado y el estudiantado; en suma, cómo se relacionan maestros y maestras, niños y niñas con el contenido de enseñanza y aprendizaje dentro de un salón de clases.

Palabras clave. Mecanismos de influencia educativa, interactividad, enseñanza, aprendizaje, intersubjetividad, participación guiada, secuencia didáctica (Tesauro Unesco).

Abstract. This article makes explicit the sense and the meaning of the qualitative investigation for the human being's life since it makes possible that from the social contexts, the actors and their inner interpret, understand and explain social phenomena. Considering the education as a social fact, in its inside takes place methodological and epistemological contributions created by Professor César Coll with the method denominated "Mechanism of Educational Influency», that strengthen the socio cultural approaches and contribute to the analysis of the educational practices, the academical context and gives specific response to which are the mechanism of educational influences that operates in the interaction between teacher and student; basically in how teachers and children relate with the education contents and the way of learning inside the classroom.

Keywords. Mechanisms of the education and its influences, interactivity, teaching, learning, intersubjectivity, participation, guided didactic sequences (Thesaurus Unesco).

Resumo. Este artigo explicita o sentido e o significado da investigação qualitativa para a vida do ser humano que possibilita que a partir dos 
contextos sociais, os atores e suas intersubjetividades interpretem, compreendam e expliquem fenômenos sociais. Considerando a educação como um feito social, em seu interior abordam-se os aportes metodológicos e epistemológicos realizados por César Coll com o método denominado «mecanismos de influência educativa», que robustecem os enfoques socioculturais e contribuem para a análise das práticas educativas, os contextos escolares e dão resposta específica a quais são alguns dos mecanismos de influência educativa que operam na interatividade entre o professorado e a estudantada; em suma, como se relacionam mestres e mestras, meninos e meninas com o conteúdo de ensino e aprendizagem dentro de uma sala de aula.

Palavras chave. Mecanismos de influência educativa, interatividade, ensino, aprendizagem, intersubjetividade, participação guiada, sequência didática (Tesaurus Unesco).

\section{Introducción}

A finales del siglo XIX, dados los acontecimientos originados al interior de la cultura, así como la disposición y el deseo de investigar para comprender e interpretar los comportamientos de los seres humanos a través de investigaciones de orden cualitativo, toma fuerza y colma diversos ámbitos sociales y culturales el interés por el estudio de las ciencias del ser humano.

Atendiendo a que algunas conductas humanas, no son viables de registrarse y elevarse bajo instrumentos y resultados de los análisis caracteríticos en las ciencias exactas, se hace necesaria la instalación de un análisis dialéctico entre el desarrollo de los seres humanos y los elementos multifactoriales tanto naturales como culturales que inicien en su proceso de formación.

La investigación cualitativa emerge como resultado de reflexiones de este tipo, y con ello por la orden social perentoria de comprender esa realidad a través de las opiniones, apreciaciones, imaginarios, representaciones sociales y formas particulares de mirar de los actores de un contexto específico, así como de la ruptura con la idea que las relaciones interculturales, el universo y el cosmos funcionan de la misma manera. 
Al respecto afirman Denzin y Lincoln (1994):

La investigación cualitativa tiene un enfoque multi-metodológico, que implica un enfoque interpretativo y naturalista a su objeto de estudio. Esto significa que los investigadores cualitativos estudian las cosas en sus ambientes naturales, intentando darles sentido e interpretando los fenómenos en función de los significados que las personas les otorgan. La investigación cualitativa involucra el estudiado uso y recopilación de una variedad de materiales empíricos (estudio de caso, experiencia personal, introspectiva, historia de vida, textos observacionales, históricos, interactivos y visuales), los cuales describen momentos y sentidos rutinarios y problemáticos en la vida de los individuos (p. 2).

Se plantean, entonces, enfoques hermenéuticos y fenomenológicos dando espacio a investigaciones referidas a las prácticas sociales, las relaciones intercisas e intrínsecas; construyendo el conocimiento a través de la indagación de una realidad múltiple, compleja, históricamente construida la cual se puede conocer, analizar, interpretar, describir, comprender y transformar a través de las acciones de los actores que la representan.

A partir de la filosofía fenomenológica de Husserl (1986), de enfoque postpositivista comprensivo hermenéutico que se gesta a fínales del siglo XIX y que alcanza su pleno desarrollo en los años 50 y 60 del siglo XX con Dilthey (1994), Husserl (1986) y Weber (2009) entre otros, se logra un desprendimiento del «método científico» para explicar el por qué y las causas de los fenómenos; se plantea la dualidad entre la metodológica explicativa que da cuenta de los fenómenos originados desde las ciencias exactas a través de deducciones y de hipótesis verificables mediante la observación instalada hasta finales del siglo XIX y la corriente metodológica por la comprensión de hechos, donde el propósito fundamental ya no es dominar y someter el medio sino comprender y buscar los significados que surgen de una situación para darle un tratamiento como un deber ser y alejarse del control rígido y restringido. Autores como Wittgenstein (1953), Kuhn (1962), Feyerabend (1995), Lakatos (1993) y Popper (1997), entre otros, así como diferentes trabajos realizados desde de la psicología Gestalt, desde el Estructuralismo Francés y del enfoque Sistémico permiten robustecer los elementos tanto teóricos como de análisis de las investigaciones de corte cualitativo. 
El enfoque hermenéutico producto del desarrollo de la filología y de la retórica, de lo bíblico o la interpretación doctrinal de pensamientos o de la interpretación y comprensión de textos, se produjo desde una trasposición al campo de lo filosófico. Dilthey (1994), fundamenta la validez de la interpretación y comprensión histórica de la realidad, en la que los significados, los sentidos, las subjetividades y la sensibilidad de los sujetos son llamados para el análisis de un fenómeno; las situaciones experienciales y observacionales son amplias para lograr correlacionar las teorías con las descripciones y comprender las realidades y su mundo simbólico. El comportamiento humano es el mismo, es sujeto y objeto de una investigación que puede afectar al fenómeno o el fenómeno lo puede afectar a él; los significados de algunas construcciones sociales, son susceptibles a los acontecimientos universales, locales y propios de una cultura que lleva implícita un cúmulo de normas y valores específicos.

La investigación de enfoque hermenéutico se sitúa no solo como una posibilidad metodológica sino como una concepción epistemológica, en la cual la existencia se descifra como una realidad social, determinada por las múltiples interpretaciones, subjetividades e intersubjetividades. Según Erickson (1986), el objetivo central de esta perspectiva es comprender la realidad desde su especificidad y plantea el estudio de caso como estrategia metodológica que posibilita investigar, describir y analizar contextos determinados resultado de variados agentes y causas; a decir de Coll (1990) se logran realizar «descripciones mas precisas y de validar las interpretaciones contextuales» (p. 225).

En el espacio escolar desde el anterior enfoque y marco metodológico, se da respuesta a cómo y cuándo se genera la interacción en el aula de clase y permite visibilizar el proceso de enseñanza y aprendizaje como un proceso social. Al respecto, Coll y Solé (1988), plantean que a partir de los contextos escolares es posible visibilizar la actividad constructiva del estudiantado, la guía y estructuración del profesorado, el contenido de aprendizaje y el contexto institucional, permitiendo de esta manera identificar, interpretar y comprender las actuaciones y significaciones explícitas en un contexto escolar determinado y tomar las aulas de clase, sus prácticas pedagógicas, como procesos de relaciones interpersonales y construcciones sociales que se producen en la interactividad.

Desde estas concepciones, el «Grupo de Investigación en Interacción e Influencia Educativa del Departamento de Psicología Evolutiva y de la Educación» de la Universidad de Barcelona, coordinado por el profesor 
Cesar Coll, ha desarrollado de manera amplia investigaciones acerca de como se da la interactividad entre profesorado y estudiantado alrededor de los contenidos o tareas de aprendizaje; indagaciones escolares resueltas mediante la investigación cualitativa de corte interpretativo desde una metodología de estudio de casos y bajo el método denominado «mecanismos de influencia educativa», el cual se detalla a continuación, después de estudiarlo y valorarlo como alternativo.

\title{
Fundamentos epistemológicos y metodológicos de los «mecanismos de influencia educativa» propuestos por César Coll
}

\section{Criterios, principios y decisiones metodológicas}

El Grupo de investigación del Departamento de Psicología Evolutiva y de la Educación de la Universidad de Barcelona Coll, Colomina, Onrubia, y Rochera (1995), plantean los mecanismos de influencia educativa que operan en la interactividad entre el profesorado y el estudiantado:

\begin{abstract}
El objetivo último no es, sin embargo, describir cómo enseñan los profesores o indagar por qué enseñan, como enseñan, al estilo de buena parte de las investigaciones didácticas al uso. El objetivo último, en términos un poco más concretos que los que hemos utilizado hasta ahora, es identificar, describir e intentar comprender algunos de los mecanismos mediante los cuales una persona, que actúa como agente educativo -madre, profesor, igual-, consigue incidir sobre otra persona -hijo, alumno, igual- ayudándole a construir un sistema de significados $-\mathrm{y}$, por lo tanto, a realizar una serie de aprendizajes relativos a una determinada parcela de la realidad, es decir, a un contenido concreto (p. 190).
\end{abstract}

En su interior se analizan los Mecanismos de Influencia Educativa (MIE) producidos en contextos educativos; esto es, las relaciones, su significación, su intencionalidad, los discursos y las descripciones detalladas de los hechos áulicos en torno a un aprendizaje.

Responde a un corte interpretativo en tanto busca determinar una realidad específica; identificar, describir, analizar y comprender las actuaciones del estudiantado en estrecha relación con el maestro y viceversa y su afectación dentro del proceso de enseñanza y aprendizaje. 


\section{Principios conceptuales que fundamentan este modelo de análisis}

\section{Interactividad}

Desde una posición epistemológica constructivista se reconoce al sujeto como actor principal en la construcción del conocimiento, y en el proceso de enseñanza y aprendizaje tres elementos constitutivos a saber: a) el estudiante, que aprende a través de su capacidad de construcción de conocimiento, b) el contenido o tarea u objeto de enseñanza y aprendizaje y c) el sujeto que apoya este proceso. «El papel del profesor es el de orientar, guiar y sostener la actividad constructiva del alumno proporcionándole las ayudas educativas necesarias; mediando entre los saberes o contenidos de aprendizaje y la actividad del alumno. Los contenidos, por su parte, mediatizan la actividad conjunta de profesores y alumnos en el proceso de construcción» (Mauri, Coll y Colomina, 2005, p. 92).

Esta triada se evidencia en la interactividad originada en las relaciones que se instituyen entre el estudiantado, el contenido y el maestro o la maestra; Coll y Onrubia (1995), afirman que la interactividad es la articulación de las actuaciones (lo que dicen y hacen) entre profesorado y el estudiantado en torno a un determinado contenido o tarea específica, es decir, los procesos interpsicológicos que subyacen a la actividad conjunta profesoradoestudiantado (Colomina, Onrubia y Rochera, 2001) y la situación de un modelo jerárquico y lineal de relación entre la conducta del niño o la niña y la de las personas adultas. Este proceso de encuentro de saberes, sentires e intersubjetividades es afectado tanto por aspectos comunicativos como por un contexto que se construye y deconstruye gracias a los procesos mentales de los actores de un escenario.

Edwards y Mercer (1984) aportan ideas importantes alrededor del contexto como un escenario tangible e intangible originado a partir del encuentro escolar.

Hay que concebir el contexto como algo mental más que lingüístico o situacional, es decir, como una propiedad de las comprensiones generales que surgen entre las personas que se comunican, no como una propiedad del sistema lingüístico que utilizan o de las cosas que se han hecho y dicho realmente, ni tampoco de las circunstancias físicas en que dichas personas se encuentran (...). Podemos decir que el proceso 
de la educación, en la medida que tiene éxito, consiste en gran parte en el establecimiento de esos «contextos mentales compartidos», de comprensiones conjuntas entre maestro y alumnos que les permite realizar juntos el discurso educacional (p. 84).

Siguiendo a Coll (2004), el análisis de la interactividad tiene lugar en:

a. La dimensión temporal, esto es, determinar el momento en que las actuaciones en el proceso de enseñanza y aprendizaje se dan ya que tanto las actuaciones o comportamientos que se presentan como semejantes pueden tener significado diferente lo que permite al maestro o la maestra realizar la intervención pertinente en el momento que lo indique la actividad o la situación.

b. La definición de las unidades de análisis que posibilita reconocer los segmentos de actividad conjunta que se aplican en el análisis de la interactividad. Para el análisis de la interactividad se elige la secuencia didáctica como unidad básica para la observación, el análisis, interpretación y comprensión de los hechos áulicos y el proceso donde se incluye la planificación, el desarrollo y la evaluación.

c. El contenido y/o tarea alrededor de la cual los participantes se movilizan y donde se origina la interdependencia entre los contenidos y la forma como el estudiantado organiza la actividad conjunta. La actividad conjunta y sus estructuras se conforman en la medida en que la interactividad, la interrelación entre el profesorado, los y las estudiantes giran alrededor de un pretexto pedagógico, es decir, en la medida en que se construye la secuencia didáctica y sus secciones de interactividad.

De igual manera, Coll y Onrubia (2001), proponen que para el análisis de la interrelación de la triada pedagógica y didáctica, es preciso detenerse tanto en el marco social- significados supuestamente compartidos- como en el marco referencial -significados compartidos introducidos dentro de la secuencia didáctica.

Por su parte, Rogoff (1993), manifiesta que en la interactividad emerge la mediación intersubjetiva como una acción que ayuda a los individuos a percibir e interpretar su medio. Una persona, el mediador, ayuda a otro a reconocer los rasgos significativos de su entorno ya sea físico o social de la experiencia inmediata o de la pasada; así, el maestro y la maestra utilizan diferentes medios de carácter situacional, instrumental o lingüístico para 
la construcción de la intersubjetividad. Este rasgo conduce al concepto de mediación semiótica, término introducido por Wertsch (1989) quien plantea que la forma que adquiere mayor relevancia en la mediación semiótica para el aprendizaje es el lenguaje.

\section{Mecanismos de Influencia Educativa}

Coll (1988) define los mecanismos de influencia educativa como aquellos procedimientos mediante los cuales los maestros o las maestras consiguen promover y facilitar en las niñas y los niños el proceso de construcción de significados y la atribución de sentido en el transcurso de las actividades escolares de enseñanza y aprendizaje. Cuando estos mecanismos son eficaces, la ayuda del profesorado es ajustada en forma constante para sintonizarse con el proceso de construcción de significados que adelanta el estudiantado.

Este autor plantea dos grandes tipos de mecanismos que posibilitan el ajuste de la ayuda del adulto al niño y la niña en el transcurso de la interacción:

La construcción progresiva de sistemas de significados, compartidos entre las personas adultas y los y las niñas y dado alrededor de una tarea o contenido como resultado del proceso de aprendizaje donde se establecen acuerdos sociales frente a los significados de los enunciados en un contexto específico y se generan negociaciones implícitas o explícitas entre los participantes.

Acerca de los acuerdos necesarios que deben darse entre los interlocutores para llegar a la construcción de sistemas de significados compartidos, se plantea que cada interpretación de un enunciado está sustentado en el desarrollo cognitivo individual y que desde allí se instaura el sistema de interpretación de significados que luego coloca en juego con otro interlocutor para constituir significados compartidos.

Con relación a las negociaciones de significados, Wertsch (1989) argumenta que son posibles cuando los interlocutores comparten en algún aspecto las definiciones de la situación, hecho que puede establecerse en diferentes niveles. Una definición de la situación es el modo en que un escenario o contexto es representado por quienes operan en él, en este evento cada participante construye e incluye metas diferentes sobre los pasos a realizar respecto a la tarea o contenido objeto de interacción; la situación también implica la representación particular de los objetos del entorno y las acciones 
de manera que la persona adulta, el niño y la niña definen como secuenciar sus acciones para conseguir sus metas o propósitos personales.

En el contexto de interacción, cuando la persona adulta ayuda a aproximarse al niño o a la niña a la zona de desarrollo próximo, según Werstch y Vigotsky (1998), surge la intersubjetividad donde coexisten tres definiciones de la situación: primero, las de cada persona adulta, niño y niña, segundo, la representación de la situación, y tercero, la comunicación que es posible entre ellos. Dentro de la relación intersubjetiva sobresale la asimetría como factor determinante dentro del proceso de construcción de sistemas de significados entre un adulto, un niño y una niña; a decir de Coll y Onrubia (1992), esta se ocasiona ya que el cambio en el adulto es temporal en tanto se da durante el proceso de aprendizaje mientras que en el niño y la niña en el aprendizaje conforma nuevas estructuras que permanecen por un período prolongado o definitivo.

La cesión y el traspaso progresivo del control y la responsabilidad. Este planteamiento desarrolla la idea de conversión de la zona de desarrollo potencial en real trazada por Vygotsky (1979), la cual se centra en las fases y mecanismos de transición desde la resolución del problema (con la ayuda de otra persona a su realización independiente y de la apropiación de los conocimientos y competencias necesarias para ello) hasta el proceso de interiorización o de transición desde el plano de la interacción social al funcionamiento intrapsicológico.

Bruner y sus colaboradores Rogoff (1990) y Wertsch (1989) trabajan sobre la noción de mecanismos de influencia educativa construida a través de la idea metodológica de andamiaje (scaffolding) elaborada por Wood, Bruner y Ross (1976), referida a las estructuras de quien participa en una construcción, creada en torno a ella, para sostenerla mientras la construye. La metáfora del andamiaje de Bruner (1998), plantea que los maestros y las maestras son quienes hacen y sostienen una estructura y con ese apoyo los niños y niñas construyen su conocimiento. A través de este proceso se favorece el aprendizaje al dirigir las intervenciones educativas más allá de los niveles de desarrollo que presenta (zona de desarrollo próximo) y por otro lado, contribuye al fortalecimiento de su autonomía en tanto que la representación del maestro y la maestra significa solo una guía para el niño y la niña, apoyándolos (andamiaje) para que posteriormente se desenvuelvan por sí mismos. 
Otro aporte importante al estudio de cesión de traspaso es la participación guiada, según Rogoff (1990), que hace referencia a las interacciones didácticas. Ésta se centra en los sistemas de actividades compartidas y pone de relieve las redes de comunicación verbal y no verbal entre el aprendiz y aquellos que interactúan así como también el grado de ajuste en la relación que mantienen. En la participación guiada el estudiantado desarrolla un papel eminentemente activo y las personas adultas son guías que ayudan a la comprensión de fenómenos o la realización de tareas un poco más complejas de las que dominan.

Por su parte Wertsch (1988) considera que a través de la intersubjetividad los interlocutores comparten algún aspecto de sus definiciones de la situación -modo en que el escenario o contexto es representado por quienes operan en él- hecho que puede establecerse en diferentes niveles: a) en el primer nivel, la definición de la situación de los niños y niñas es tan diferente a la de las personas adultas que resulta difícil la comunicación, b) en el segundo nivel de intersubjetividad comienza a producirse una definición compartida, c) en el tercer nivel la niña y el niño ya pueden realizar inferencias para comprender las producciones reguladas por las personas adultas, incluso cuando no sean explícitas, d) en el cuarto y último nivel la niña y el niño asumen la responsabilidad sobre la realización de la tarea.

De la misma manera, el autor analiza algunos mecanismos semióticos que se producen en el curso de la interacción y que operan en la zona de desarrollo próximo utilizados por las personas adultas para desarrollar las competencias individuales y la construcción de intersubjetividad de los y las niñas; esto permite la aparición de nuevos procesos, capacidades y reorganizaciones en el conocimiento los cuales rápidamente se reflejan en cambios explicitados en las diversas formas de interacción social, los que a su vez le permiten construir progresivamente niveles de intersubjetividad más complejos.

Coll y Onrubia (2001), interesados en los procesos y mecanismos involucrados en la influencia educativa plantean que los usos de habla posibilitan que los agentes educativos o miembros más competentes en la interacción, ayuden -asistan y orienten- a los aprendices o miembros menos competentes en la construcción de sistemas de significados cada vez más ricos y culturalmente válidos sobre distintas parcelas de la realidad, sostiene que el discurso tiene un papel preponderante en 
la construcción de los saberes, más específicamente en la construcción de significados compartidos entre el profesorado y los estudiantes en situaciones de aula.

\section{Decisiones metodológicas}

Por ser una metodología aplicada al campo de la educación se abordan dos aspectos: primero la identificación de las secuencias interactivas que se dan dentro del proceso de construcción de un conocimiento y desarrollo de la competencia y segundo como la interacción entre el maestro y la maestra y el estudiantado influye en la actividad autoestructurante de los y las estudiantes.

Se utilizan los mismos procedimientos tanto para la recolección de los datos como para el análisis de los mismos, en tres fases:

a. Fase de elaboración en la cual se define y elabora la tipología de instrumentos a aplicar.

b. Fase de recolección de datos, donde se recogen, se organizan y se revisan los instrumentos aplicados. Se incorporan instrumentos que corresponden a cada secuencia didáctica específica, mediada por el contexto donde se desarrollen; se enuncian las siguientes:

Entrevistas iniciales con los maestros para determinar las unidades a observar en su propuesta de trabajo (planificación, evaluación inicial), y las argumentaciones que hacen los niños y las niñas en la secuencia didáctica.

Registros escritos narrativos de videos o audio realizados durante las observaciones de las secuencias didácticas así como transcripciones literales de las verbalizaciones de los actores.

Entrevista final con los maestros sobre la secuencia didáctica y la contrastación de su desarrollo con respecto a lo planificado, evaluación sobre las argumentaciones construidas por los y las estudiantes después de la secuencia didáctica.

Recolección de registros escritos como: El proyecto Educativo Institucional, proyectos y planes de aula. 
Las secuencias de enseñanza y aprendizaje se observaran de principio a fin incluyendo por supuesto las competencias y los niveles de desempeño que busca desarrollar en el estudiantado, las actividades realizadas dentro del proceso de enseñanza y aprendizaje y la evaluación que se den aunque sea en sesiones distintas de la misma secuencia.

Se recogen los datos a partir de distintas fuentes y técnicas como entrevistas, observación, análisis de documentos a través de las cuales se intenta describir en detalle la actividad conjunta dada al interior de una secuencia didáctica

c. Fase de análisis de datos y concreción definitiva de los instrumentos de evaluación. Desde el modelo de análisis de la interactividad trabajada por Coll y los miembros de su grupo de investigación (Col, 2004; Coll, Onrubia y Mauri, 2008 y Onrubia, 2005), se exploran la dimensión temporal, las exigencias y condicionantes impuestas por la naturaleza del contenido y/o la estructura de la tarea, cómo se organiza la tarea conjunta, la forma integrada de la actividad discursiva y no discursiva de los participantes y la actuación de estos en el flujo en que se inscribe dicha actuación.

La investigación y su análisis recae básicamente sobre campos -momento y espacio donde ocurre la interactividad del aula de clase- en los que el objeto de investigación a decir de Coll, son las formas de actividad conjunta entre maestras, niños y niñas en torno a una tarea o actividad conjunta de aprendizaje.

En los campos se visualizan elementos como: la influencia de la interactividad en el desarrollo de la construcción del conocimiento de los niños y las niñas, las formas de actividad conjunta dadas entre las maestras, los maestros y el estudiantado donde se intenta afectar el conocimiento en los y las infantes, determinadas por variables -conocimientos iniciales de los niños y niñas, prácticas pedagógicas relevantes, conocimientos previos que poseen alrededor de una tarea que posibilita la explicitación de las concepciones iniciales y la creación de otras más complejas, conectadas con las anteriores-, las formas de actividad conjunta que contribuyen al estudiantado en el dominio autónomo y autoregulativo de la construcción del conocimiento y la interacción de los niños y las niñas cuando se encuentran trabajando colaborativamente y su ayuda a la construcción de conocimiento. 
Para su desarrollo se tienen en cuenta las decisiones metodológicas básicas planteadas por (Coll, Colomina, Onrubia y Rochera, 1992), descrito en niveles y unidades de análisis del Modelo para el análisis de la interactividad (Coll y Onrubia, 1992a, 1992 b) en el que se plantean dos niveles de análisis: formas de organización de la actividad conjunta, es decir cómo evolucionan las formas de actuación de los participantes y como construyen progresivamente significados compartidos.

El análisis de la interacción determina niveles con objetivos específicos que los diferencian pero que se encuentran íntimamente relacionados; se definen los niveles de análisis específicos e interconectados que forman un sistema de conjunto:

Primer nivel de observación y análisis. Desde este nivel se espera caracterizar la interacción, la organización de la actividad conjunta la cual se «centra en la articulación de las actuaciones o comportamientos del profesor y de los alumnos en torno a la tarea o contenido de aprendizaje y su evolución a lo largo de la secuencia didáctica» (Coll y Onrubia, 1992); en este nivel se develan «los dispositivos vinculados a los procesos de cesión y traspaso del control y la responsabilidad» (Onrubia, 1993, p. 89).

Una de las unidades de primer orden de este macro nivel es la Secuencia Didáctica (SD) -Coll y Onrubia (1992b) plantean que es posible no solo recoger datos sino también analizarlos e interpretarlos-.

La Secuencia Didáctica. Como unidad de observación se define en función de la preocupación por el flujo temporal en los procesos de enseñanza y aprendizaje, es a su vez el proceso completo de enseñanza y aprendizaje que incluye los componentes del mismo (competencias, indicadores de desempeños, unidades temáticas, actividades y tareas de enseñanza y aprendizaje y evaluación). Coll (1988), citado por Coll, Colomina, Onrubia y Rochera (1992), la define como:

Un proceso de enseñanza y aprendizaje en miniatura, esto implica la necesidad de identificar o inferir los principales componentes de un proceso de enseñanza y aprendizaje(...): objetivos educativos concretos, utilización de un determinado material, determinadas actuaciones del enseñante cuyo destinatario es el alumno, determinadas actuaciones del alumno sobre el material en torno a los objetivos y contenidos propuestos por el enseñante, determinadas expectativas del enseñante, a propósito de las 
actuaciones del alumno, posibilidad de proceder a una evaluación de las actividades del alumno en función de las expectativas del enseñante. Así pues, para poder hablar de una secuencia didáctica, tendremos que identificar inequívocamente su inicio, su desarrollo y su finalización (p. 27).

Las Sesiones (S). Acerca de las sesiones o escenarios de actividad, Onrubia plantea que en su interior «suponen cortes en el tiempo en desarrollo de la $\mathrm{SD}$, proporcionan información relevante sobre el manejo por parte de los participantes de las discontinuidades temporales en la actividad» (Onrubia, 1993, p. 90).

Segmentos de interactividad (SI). Según Coll y Onrubia (1996) determinados por los siguientes criterios: a) la estructura de participación social que rige las obligaciones y los derechos comunicativos de los participantes (Quién habla, qué hace con su intervención); b) la estructura de la tarea académica (contenido); c) la finalidad o intencionalidad instruccional que preside dicha actividad. Estos segmentos de interactividad tienen secuencias definidas a partir de la unidad temática o del contenido y la estructura de participación; así mismo según Onrubia (1993) determinan que hacen y dicen los participantes dentro de un momento específico de la actividad conjunta.

Los segmentos de interactividad (SI) en la unidad de análisis son formas específicas de organización de la actividad conjunta en el interior de las sesiones, caracterizadas por determinados patrones de actuaciones articuladas del profesor y de los alumnos así como por una cierta cohesión temática interna. Definen qué pueden hacer y decir los participantes en un momento dado de la actividad conjunta, y pueden cumplir funciones instruccionales particulares (Coll y Onrubia, 1994).

Algunos elementos del análisis de las unidadesson los siguientes: identificación y descripción de los segmentos de interactividad (SI); identificación y descripción de los patrones de actuación dominantes de los participantes para cada tipo de segmento de interactividad; identificación y descripción, si existen, de las configuraciones de segmentos de interactividad (SI); interpretación estática, desde el punto de vista del ejercicio de la influencia educativa, de los SI, los patrones de actuación y los CSI identificados: funciones instruccionales de los SI y los patrones de actuación identificados, acumulación de evidencia convergente y análisis de casos discrepantes; análisis de la evolución 
de los SI y CSI (presencia/ausencia, frecuencia y distribución a lo largo de las sesiones y del conjunto de la SD), elaboración de mapas de interactividad del conjunto de la SD, análisis de la evolución de los patrones de actuación dominantes para cada tipo de SI. Interpretación dinámica, desde el punto de vista del ejercicio de la influencia educativa, de la evolución de los SI, CSI y patrones de actuación: cambios en las funciones instruccionales de los SI y en los patrones de actuación identificados, acumulación de evidencia convergente y análisis de casos discrepantes; interpretación de conjunto del primer nivel de análisis.

El análisis de cómo surgen y evolucionan los SI y de los ajustes que se producen en éstos, permiten construir la unidad de análisis de segundo orden del primer nivel: las configuraciones de segmentos de interactividad (CSI). Admiten colocar en relación a los (SI), cuando regularmente uno o varios (SI) se presentan seguidos unos de otros en distintos momentos de la secuencia didáctica.

El nivel intermedio de observación y análisis de la interacción donde se determinan los recursos, estrategias, formas de actuación de las maestras y de los estudiantes alrededor de una tarea y en relación con los otros.

Segundo nivel de análisis. Centrado en el significado que los participantes negocian y construyen gracias a su actividad discursiva. Desde este nivel se detecta cómo y cuándo se produce las construcciones progresivas de sistemas de significación compartidas, en este nivel se plantean preguntas como: ¿̇cuáles son, cómo se presentan y cómo se organizan los significados durante la SD?; íqué entidad debe ser objeto directo de indagación para captar los significados que actualizan los participantes mediante su actividad discursiva?

En suma, el análisis hace acento en la negociación de significados y representaciones de los actores en una actividad conjunta escolar y en un contexto específico.

Los mensajes. Una de las unidades de análisis de primer orden a abordar en este nivel son los «mensajes», considerados como las expresiones mínimas con significado en su contexto, enunciadas por cualquiera de los participantes en la actividad conjunta. Onrubia define el mensaje como «la expresión, a cargo de uno de los participantes en la actividad conjunta, de una unidad de información que tiene sentido en sí misma 
en su contexto de enunciación, y que por lo tanto puede descomponerse, sin perder su potencialidad comunicativa en el contexto de la actividad global en que ha sido producida» (Onrubia, 1992, p. 225). Los mensajes especifican los aspectos que permiten identificar los significados que estos contienen, definir los términos y procedimientos apropiados para llevar a cabo un análisis de supra-mensajes.

El análisis intramensajes se realiza desde dos aspectos: desde el contenido referencial y desde su fuerza ilocutiva, el primer aspecto implica el análisis del contenido inferencial de los mensajes; es decir, identificar sobre lo que hablan los mensajes, las entidades objetos, acciones, situaciones entre otros sobre lo que hablan los participantes en una actividad conjunta; o sea, las actividades que actualizan los participantes mediante su actividad discursiva y que constituyen un aspecto del significado de los enunciados; el segundo aspecto hace referencia a la fuerza ilocutiva que poseen los significados, lo ilocutorio de los significados está relacionado con lo que Austin et al. (1976) denomina «los actos de habla» que llevan implícitos una acción, donde los mensajes son analizados en su totalidad, desde su fuerza ilocutiva.

Desde este nivel se analizan los significados que se construyen durante una secuencia didáctica, es decir, se identifican las estrategias discursivas y mecanismos semióticos que utilizan tanto el profesorado como el estudiantado cuando hablan y como negocian y co-construyen los significados y los sentidos alrededor de un objeto de estudio.

Configuraciones de mensajes (CM). Las unidades de análisis de segundo orden del segundo nivel son las configuraciones de mensajes (CM), las cuales son «agrupaciones de mensajes que, por su organización y estructuración, trasmiten significados que no pueden reducirse a la suma de los significados trasmitidos por cada uno de los mensajes que las integran» (Coll, Colomina, Onrubia y Ranchera, 1995, p. 59 ).

Son unidades de análisis derivadas o de segundo orden las siguientes: identificación de los mensajes; identificación y caracterización de los significados que se negocian y co-construyen a través de los mensajes (M), y de algunas características de la manera en que se presentan y re-presentan esos significados; identificación de ámbitos referenciales e ítems de contenido, e identificación de fuerza ilocutiva de los Mensajes y enunciados; identificación y descripción de configuraciones de mensajes (CM), si es que existen: estructuras temáticas, estructuras 
conversacionales, etc.; interpretación estática, desde el punto de vista del ejercicio de la influencia educativa, de los significados que se negocian y co-construyen a través de los $\mathrm{M}$, y de algunas características de la manera en que se presentan y re-presentan esos significados; mecanismos semióticos implicados en la presentación de los ítems de contenido, funciones instruccionales de las configuraciones de mensajes, acumulación de evidencia convergente, y análisis de casos discrepantes; análisis de la evolución de los significados que se negocian y co-construyen a través de los M, y de algunas características relacionadas con la manera en que se presentan y re-presentan esos significados: tendencias evolutivas en los $\mathrm{M}$ y en las CM; interpretación dinámica, desde el punto de vista del ejercicio de la influencia educativa, de la evolución de los significados que se negocian y co-construyen a través de los M, y de algunas características acerca de la manera en que se presentan y re-presentan esos significados; cambios en los mecanismos semióticos implicados en la presentación del contenido tanto en el ámbito de los $\mathrm{M}$ como de las CM, acumulación de evidencia convergente, y análisis de casos discrepantes; interpretación de conjunto del segundo nivel de análisis; interpretación global integrada del primer y del segundo nivel de análisis.

Mapa de Interactividad. El análisis de la interactividad permite identificar y describir los dos grandes mecanismos de influencia educativa que operan en los procesos de enseñanza aprendizaje y, presumiblemente, en cualquier interacción adulto-niño de un carácter educativo: «un proceso de construcción progresiva de sistemas de significados compartidos cada vez más ricos y complejos entre profesores y estudiantes y un proceso de traspaso progresivo del control del adulto hacia el niño. Ambos mecanismos compartirán algunas características comunes»(Colomina, 2001).

Paralelamente a este proceso de análisis es preciso construir el mapa de Interactividad desde el cual se describe y caracteriza la estructura de la actividad conjunta dentro de una secuencia didáctica y la comprensión tanto de la interacción como de los mecanismos de cesión y traspaso progresivos de la responsabilidad y el control en el aprendizaje.

Coll (1990) plantea que el mapa de interactividad «ofrece una visión de conjunto de las distintas formas de organización de la actividad conjunta que aparece en las secuencias didácticas de su distribución temporal y de su evolución en el transcurso de las sucesivas sesiones que las conforman» (p. 225). 


\section{Reflexiones finales}

El modelo de mecanismos de influencia educativa permite identificar, describir, interpretar y comprender los mecanismos interpsicológicos que se dan en la interacción entre el estudiantado y la influencia educativa que el maestro y la maestra plantean para orientar en la construcción del conocimiento en torno a una tarea o contenido.

El estudio y la apropiación de los conceptos sobre interactividad y mecanismos de influencia educativa son nucleares a la hora de realizar un análisis de las prácticas educativas del aula. Desde estos conceptos se puede identificar cuales son las formas en que se organiza la actividad conjunta: las formas de organización de la actividad conjunta, las estructuras de participación que subyacen a esas formas y los patrones de actuaciones de los participantes que las configuran y los significados negociados por los participantes en el marco de esa estructura de actividad, es decir, qué y cómo se habla en un contexto definido.

El modelo de análisis de los mecanismos de influencia educativa lleva consigo el análisis de las secuencias didácticas, las que son concebidas como unidades básicas del registro, análisis e interpretación; así mismo, éste lo hace sobre dos pretextos pedagógicos, uno la interactividad y el otro la construcción de significados, los cuales también son analizados a la luz de una unidad específica.

Es importante reconocer en el estudio de las prácticas educativas del aula la aplicación de cuestionarios, entrevistas, e instrumentos que hacen parte del análisis en situaciones planificadas como también y dado que dentro de el aula se originan intercambios verbales y no verbales, la implementación de metodologías que explicitan y dan cuenta sobre las actuaciones y la participación de agentes educativos dentro del proceso de construcción del conocimiento, en las que se pueden identificar secuencias, patrones de actuación estructuras de participación, los elementos de control y trasferencia así como los mapas de interactividad y la relación entre ellos.

Esta opción investigativa en el ámbito escolar, dadas sus particularidades, exige la ruptura con modelos de investigación y transferencias no funcionales, que partan del análisis de su realidad y el planteamiento de los problemas y objetivos, la selección de técnicas para recopilar información la codificación y clasificación de datos, el análisis e 
interpretación de resultados de tal manera que se elaboren teorías que respondan en un espacio crítico y en consecuencia de progreso, en las que la práctica pedagógica se comparta, analice y evalúe con los agentes educativos directos, donde se replanteen las relaciones dadas al interior del aula, se inventen nuevas formas de comunicación.

\section{Referencias}

Austin, J. Davidson, D. Danto, A., et al. (1976). La filosofía de la acción. Madrid: Fondo de Cultura Económica.

Bruner, J. (1998). Realidad mental y mundos posibles. Barcelona: Gedisa.

Coll, C. (1988). Actividad conjunta y habla: una aproximación al estudio de los mecanismos de Influencia educativa. En: Coll, C.; Colomina, R.; Onrubia, J. y Rochera, M.J. (1992). Infancia y Aprendizaje, 59/60:189-232.

Coll, C. (1990). Un marco de referencia psicológico para la educación escolar: la concepción constructivista del aprendizaje y la enseñanza. En: C. Coll, J. Palacios y A. Marchesi (Comps), Desarrollo psicológico y educación II. Psicología de la Educación. Madrid: Alianza, p. 225

Coll, C. (2004). Psicología de la educación y prácticas educativas mediadas por las tecnologías de la información y la comunicación: una mirada constructivista. Sintética. 25,1-24

Coll, C., Onrubia, J. y Mauri, T. (2008). Tecnología y prácticas pedagógicas: las TIC como instrumentos de mediación de la actividad conjunta de profesores y estudiantes. En Anuario de Psicología, 38(3), 377-400.

Coll, C. y Onrubia, J. (1992 a). El análisis del discurso y la construcción de significados compartidos en el aula. En: Revista Latina de Pensamiento y Lenguaje, 2, 241-259.

Coll, C. y Onrubia J. (1992b). La dimensión temporal y los procesos interactivos en las actividades de enseñanza y aprendizaje: Un desafío teórico y metodológico. Comunicación presentada en la I Conference for Socio-Cultural research, Madrid, 15 - 18 september.

Coll, C. y Onrubia J. (1994). Temporal dimension and interactive processes in teachig/ learning activities: A theoretical amb methodological challenge. En: Mercer, N. y Coll, C. (eds.). Explorations in socio- 
cultural studies. Vol. 4: Teaching, learning and interaction. General Editors: P.del Río, A. Álvarez y J. Wertsch.

Coll, C. y Onrubia J. (1995). Discusión entre alumnos e influencia educativa del profesor. En Revista: Textos No. 20. Barcelona: Grao.

Coll, C. y Onrubia J. (2001). Estrategias discursivas y recursos semióticos en la construcción de sistemas de significados compartidos entre profesor y alumnos. En: Investigación en la Escuela, 45.

Coll, C. y Solé, I. (2001). Enseñar y aprender en el contexto del aula. En: C. Coll, Palacios y A. Marchesi (Comps), Desarrollo psicológico y educación.Vol.II. Psicología de la Educación. Madrid: Alianza.

Colomina, R. (2001). Activitat conjunta i influència educativa en el context familiar.

Colomina, R., Onrubia, J. y Rochera, Ma J. (2001). Interactividad, mecanismos de influencia educativa y construcción del conocimiento en el aula. En: C. Coll., J. Palacios y A. Marchesi, (Comps.). Desarrollo psicológico y educación. 2. Psicología de la educación escolar (pp. 437-458). Madrid: Alianza.

Denzin, N. K. y Lincoln, Y. S. (1994). Introduction: Entering the Field of Qualitative Research y The Fifth Moment. En: Denzin, N. K., Lincoln (eds.). Handbook of Qualitative Research. California: Sage Publications.

Dilthey, W. (1994). Introducción a las ciencias del espíritu. México: Editorial Fondo Cultura Económica.

Edwards, D. y Mercer, N. $(1994,1998)$. El conocimiento compartido. El desarrollo de la comprensión en el aula. Madrid: Paidós.

Erickson, F. (1986). Qualitative methods in research on teaching. En: Wittrock, M.C. (Ed.), Handbook of research on teaching (pp.119161). New York: Macmillan.

Feyerabend, P. (1995). Tratado contra el método. Madrid: Tecnos.

Husserl, E. (1986). Ideas relativas a una fenomenología pura y una filosofía fenomenológica, Libro Primero: Introducción general a la fenomenología pura. Traducción de José Gaos. México: Fondo de Cultura Económica, 3a. ed.

Kuhn (1962). La estructura de las revoluciones científicas. Madrid: Breviarios del Fondo de Cultura Económica. 
Lakatos (1993). La metodología de los programas de investigación científica. Madrid: Alianza.

Mauri, T., Coll, C. y Colomina, R. J. (2005). La calidad de los contenidos educativos reutilizables: diseño, usabilidad y prácticas de uso. En: Revista de educación a distancia: http://www.um.es/ead/redM2 pp11

Onrubia, J. (1992). Interactividad e influencia educativa: aprendizaje de un procesador de textos. Tesis doctoral no publicada. Universidad de Barcelona.

Onrubia, J. (1993). Interactividad e influencia educativa en la enseñanza/ aprendizaje de un procesador de textos: una aproximación teórica y empírica. Anuario de Psicología. p. 89.

Onrubia, J. (2005). Aprender y enseñar en entornos virtuales de enseñanza y aprendizaje: actividad conjunta, ayuda pedagógica y construcción del conocimiento. En: RED: Revista de Educación a Distancia, monográfico. Consultado el 26-10-09 en http://um.es/ead/red/M2.

Popper, K. (1997). El conocimiento objetivo. Madrid: Tecnos S.A.

Rogoff, B. (1990). Apprenticeship in thinking. Cognitive development in social context. New York: Oxford University Press.

Rogoff, B. (1993). Aprendices del pensamiento. El desarrollo cognitive en el contexto social. Barcelona: Paidós.

Vygotsky, L.S. (2001) [1934]. Pensamiento y lenguaje. En Obras escogidas II. Madrid.

Vygotsky, L.S. (1979). Pensamiento y lenguaje. Buenos Aires: La pléyade.

Weber, M. (2009). La objetividad del conocimiento en la ciencia social y en la política social. Madrid España: Editorial Alianza.

Werstch, J, V. (1988). Vygotsky y la formación social de la mente. Barcelona: Paidós.

Werstch, J, V. (1988, 1989). Los mecanismos semióticos en la actividad cognitiva conjunta. Infancia y aprendizaje. 47, 3-36 y 2-22

Werstch, J. y Vygotsky. (1998). La formación social de la mente. Barcelona: Paidós.

Wood, D, Bruner, J y Ross. (1976). The role of tutoring in problem solving. Journal of Child Psychology and Psychiarty.17, 89-100. 